Research Article

\title{
Application of Multiphysical Domain Modeling in Electrical System Simulation
}

\author{
Cao Zheng, ${ }^{1}$ Zhou Yuanjun, ${ }^{1}$ and Wang $\mathrm{Na} \mathbb{D}^{2}$ \\ ${ }^{1}$ School of Automation Science and Electrical Engineering, Beihang University, Beijing 100191, China \\ ${ }^{2}$ Engineering Training Center, Beihang University, Beijing 100191, China \\ Correspondence should be addressed to Wang Na; wn1203158@163.com
}

Received 27 December 2019; Revised 1 June 2020; Accepted 24 August 2020; Published 28 September 2020

Academic Editor: Salvatore Strano

Copyright (c) 2020 Cao Zheng et al. This is an open access article distributed under the Creative Commons Attribution License, which permits unrestricted use, distribution, and reproduction in any medium, provided the original work is properly cited.

The energy structure change of more electric aircraft makes the aircraft airborne system more complicated. Each subsystem realizes the transmission, interaction, and conversion of energy and information through the dynamic coupling and coordinated control of electrical energy, mechanical energy, hydraulic energy, and thermal energy. This paper applies the multiphysical domain modeling method with the parameter identification according to the original model data. Based on the power conversion relationship of electrical equipment, it defines the port with the power potential variable and flow variable and is supplemented by the information control. It can show the dynamic characteristics, power conversion, and loss characteristics of the device itself. The models can conveniently perform the large-scale system integration, which not only can build the complex electrical equipment formed by multiphysical domain models with the series connection but also can build a complex power supply system formed by multiphysical domain models with the parallel connection.

\section{Introduction}

The secondary energy of the more electric aircraft gradually uses electrical energy to replace hydraulic energy and pneumatic energy for the aircraft energy optimization [1-3]. In the design of the aircraft electrical system, the performance of the electrical equipment in terms of energy utilization, power loss, and heat conduction needs to be taken as one of the evaluation indicators [4-6].

The electrical equipment operation of the more electric aircraft involves the transmission, interaction, and conversion of the multiphysical domain, such as electrical energy, mechanical energy, hydraulic energy, thermal energy, and information [7, 8]. Since the conversion process is dynamic, it is obviously inaccurate to discuss the energy utilization performance simply by using device efficiency, and a digital simulation method is needed $[9,10]$. The energy usage of the aircraft electrical system is related to flight missions, involving energy conversion, energy transmission, and energy consumption. Therefore, it is necessary to form the actual electrical system in the structure and perform dynamic simulation under different missions [11].

In order to realize the simulation of the electrical system multiphysical domain power conversion, the digital model of the electrical equipment is required to be established. The multiphysical domain modeling can solve the coupling problems [12]. The traditional multiphysical modeling methods are the physical structure modeling, the equivalent circuit modeling, and the analysis structural modeling. The structure of electrical equipment is more accurately represented to describe the characteristics of electrical equipment by the physical structure modeling. However, the problem is the operation speed. When the more complex system including nonlinear components is built, it will cause the computer calculation speed to be too slow in simulation.

The equivalent circuit modeling has the disadvantage because the structure is simplified. The more details and nonlinear characteristics cannot be accurately represented. The modeling workload by the analysis structural modeling is relatively large, and the nonlinear modeling is especially cumbersome. The input and output may be variables of 
different physical domains. When the electrical system integration is achieved, the system structure may be confused and inconvenient in engineering application.

The bond graph method is applied for the hydraulic system [13, 14], and the Modelica is applied for the electromechanical systems $[15,16]$. It can accurately represent its power conversion and loss characteristics when expressing the device dynamic operating characteristics [17]. In addition, because the electrical component model is used to build an electrical system, which contains a large number of nonlinear components, the transients in the simulation result in slower operation speed. Therefore, in order to achieve large-scale integrated simulation, there should be a more suitable computing speed.

This paper applies the multiphysical domain modeling method with the parameter identification according to the original model data. The analytical model based on the energy conversion process of the electrical equipment is supplemented by the information control. The model uses the energy transmission between the electrical equipment as the interface between the models, enabling the large-scale integrated electrical system simulation, and the simulation operation speed is improved.

\section{Electrical Equipment Structure and Multiphysical Domain Model}

Compared with the traditional aircraft, the increase in electrical equipment of more electric aircraft is the electrical drive equipment. The electromechanical actuator (EMA) $[18,19]$ and the electrohydraulic actuator (EHA) $[20,21]$ of the flight control system are the most typical multiphysical domain energy conversion devices. Because it presents a high-power short-term working mode, it has the greatest impact on the power characteristics of the power supply system. This paper analyzes the modeling method of the typical electrical equipment.

2.1. Structure of Typical Electrical Equipment. The electromechanical actuator is taken as an example to analyze the structural characteristics of the electrical equipment. The EMA is an electrical drive position servo control system for the aircraft surface. The physical model of the EMA is shown in Figure 1. It consists of the inverter, the brushless direct current motor (BLDCM), the reduction gear, and the microprocessor. The LC electromagnetic interference filter (EMI) is also on the power supply side.

The electrical drive equipment shown in Figure 1 is structurally divided into two parts.

2.1.1. Power Conversion Section. From the perspective of energy conversion, the electromechanical actuator performs the conversion of the electrical energy to mechanical energy, and the conversion power is determined by the fight mission. At the same time, all parts of the equipment have the power loss. It transforms parts of the electrical energy and mechanical energy into thermal energy and forms a secondary power conversion process.
2.1.2. Information Conversion Section. The main function of the information conversion part is to complete the control during the equipment. For example, the EMA realizes the closed-loop control of the rudder surface position $\theta_{a}$, the motor speed $\Omega_{m}$, and the armature current $I_{d}$. In addition, it can also monitor the running status of the device and communicate with the host computer and other devices.

The physical model shown in Figure 1 is not convenient for the large-scale integration. Because of the nonlinear components, simulation transients result in the slower operation.

2.2. Model Based on Power Conversion. According to the structure of the electrical device shown in Figure 1, the model of the electrical device can be divided into two modules: the power conversion module (PCM) and the information conversion module (SCM), as shown in Figure 2:

(1) The PCM represents the device power conversion process. The power conversion is mainly the conversion between two kinds of energy sources. The thermal energy generated by the power loss is the third additional energy conversion relationship for the performance analysis of the power loss and efficiency.

(2) The SCM function of the electrical equipment is the same as that of the information conversion part in Figure 1. The equipment operational control becomes the PCM control, and monitoring and communication status can be realized at the same time.

\section{Power Conversion Module of Multiphysical Domain Model}

3.1. Power Conversion of Multiphysical Domain. The PCM shown in Figure 2 exhibits the power conversion characteristics implemented by the electrical device, which is the power that transforms one type of physical domain power into another. For example, the generator realizes the energy conversion from mechanical energy to electrical energy, and the electrical equipment realizes the energy conversion from electrical energy to various forms of energy, including mechanical energy, hydraulic energy, pneumatic energy, and thermal energy. Therefore, the operation of the more electric aircraft electrical system is a multiphysical domain energy conversion process.

When the power conversion model shown in Figure 2 is established, the input power and output power are represented by the potential variable $Y$ and the flow variable $X$, which are defined by the connection type of the multiphysical domain modeling [13]. The power types involved in the electrical system of the more electric aircraft and the corresponding potential variables and flow variables are shown in Table 1.

3.2. Analysis Model of PCM. The PCM of the electrical equipment model can be represented as Figure 3(a). The potential variable of the input power (source power) is 


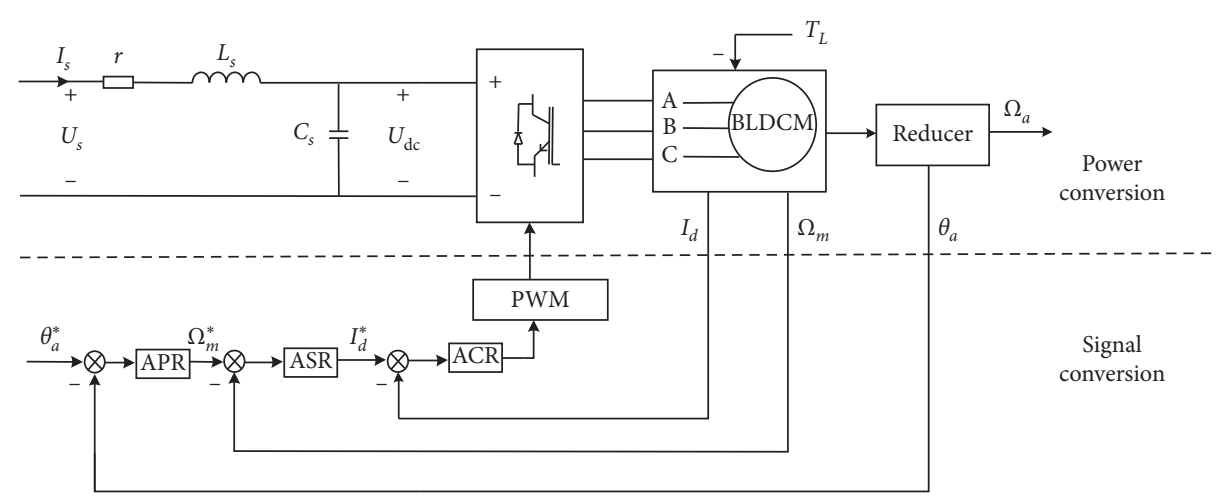

Figure 1: Principle block diagram of the electromechanical actuator.

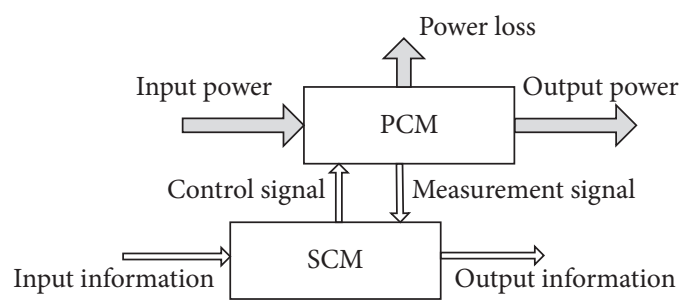

Figure 2: Model structure of the electrical equipment.

TABLE 1: Potential variables and flow variables for different power types.

\begin{tabular}{lcc}
\hline Power form & Potential variable & Flow variable \\
\hline $\begin{array}{l}\text { Electrical power } \\
\text { Rotating machine } \\
\text { power }\end{array}$ & Voltage $U(\mathrm{~V})$ & $X$ \\
$\begin{array}{l}\text { Linear machine } \\
\text { power }\end{array}$ & Current $I(\mathrm{~A})$ \\
Hydraulic power $\Omega(\mathrm{rad} / \mathrm{s})$ & Torque $T(\mathrm{~N} \cdot \mathrm{m})$ \\
Thermal power & Pressure $p(\mathrm{MPa})$ & $\begin{array}{c}\text { Moving speed } v \\
(\mathrm{~m} / \mathrm{s})\end{array}$ \\
Electrical power & Temperature $T(\mathrm{~K})$ & $\begin{array}{c}\text { Flow }\left(\mathrm{m}^{3} / \mathrm{s}\right) \\
(\mathrm{W})\end{array}$ \\
\hline
\end{tabular}

represented as $Y_{s}$, and the flow variable is represented as $X_{s}$. The potential variable of the output power (load power) is $Y_{L}$, and the flow variable is $X_{L}$. The power loss output is generally thermal power. The ambient temperature $T_{w}$ is a potential variable, and the heat flow $\Phi_{w}$ formed by the loss is a pseudo flow variable. $C$ is the control signal.

According to the relationship of the PCM input/output variables shown in Figure 3(a), the analytical method can be described as

$$
\left[\begin{array}{c}
Y_{L}(s) \\
X_{s}(s)
\end{array}\right]=\left[\begin{array}{cc}
\mathbf{W}_{1}(s) & \mathbf{W}_{3}(s) \\
\mathbf{W}_{2}(s) & \mathbf{W}_{4}(s)
\end{array}\right]\left[\begin{array}{c}
Y_{s}(s) \\
X_{L}(s)
\end{array}\right],
$$

where the transfer functions of $\mathbf{W}_{1}(s), \mathbf{W}_{2}(s), \mathbf{W}_{3}(s)$, and $\mathbf{W}_{4}(s)$ reflect the dynamic characteristics between variables in the power conversion process. The power loss of the electrical device is determined by the device operating state. Therefore, the loss model can be expressed as a function of other variables, namely,

$$
\Phi_{w}=f_{w}\left(Y_{s}, X_{s}, Y_{L}, X_{L}, T_{w}\right) .
$$

For the electromechanical actuator shown in Figure 1, the analytical formula of the PCM shown in Figure 3(b) can be described as follows:

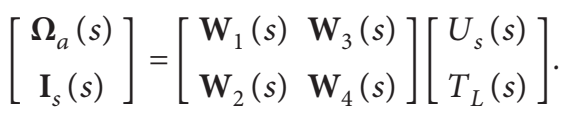

The potential variable of the input power in equation (3) is the power supply voltage $U_{s}$, and the flow variable is the supply current $I_{s}$. The potential variable of the output power is the angular velocity $\Omega_{a}$ of the aircraft rudder surface, and the flow variable is the resistance torque $T_{L}$ of the rudder surface. $D$ is the duty cycle of the PWM signal.

3.3. System Control Model of PCM. The energy conversion relationship of the entire device is shown in equation (1). As shown in Figure 2, the energy conversion process of the electrical device needs to perform under the SCM control. In order to express the power conversion relationship under the control process, the power conversion module can be rewritten as

$$
\begin{aligned}
{\left[\begin{array}{c}
Y_{L}(s) \\
X_{s}(s)
\end{array}\right] } & =\left[\begin{array}{ll}
\mathbf{W}_{1}(s) & \mathbf{W}_{3}(s) \\
\mathbf{W}_{2}(s) & \mathbf{W}_{4}(s)
\end{array}\right]\left[\begin{array}{c}
Y_{s}(s) \\
X_{L}(s)
\end{array}\right] \\
& =\left[\begin{array}{cc}
G_{1}(s) & G_{3}(s) \\
G_{2}(s) & G_{4}(s)
\end{array}\right]\left[\begin{array}{c}
y_{s c}(s) \\
x_{\mathrm{Lc}}(s)
\end{array}\right],
\end{aligned}
$$

where $y_{\mathrm{sc}}$ and $x_{\mathrm{Lc}}$ are the device control and interference variables, respectively, which are generated by the SCM and have a certain functional relationship with $Y_{s}$ and $X_{L} \cdot G_{1}(s)$, $G_{2}(s), G_{3}(s)$, and $G_{4}(s)$ are the transfer functions of the device control system.

The electromechanical actuator shown in Figure 1 is taken as an example and the power conversion module of the control system is

$$
\begin{aligned}
{\left[\begin{array}{c}
\Omega_{a}(s) \\
I_{s}(s)
\end{array}\right] } & =\left[\begin{array}{ll}
\mathbf{W}_{1}(s) & \mathbf{W}_{3}(s) \\
\mathbf{W}_{2}(s) & \mathbf{W}_{4}(s)
\end{array}\right]\left[\begin{array}{c}
U_{s}(s) \\
T_{L}(s)
\end{array}\right] \\
& =\left[\begin{array}{ll}
G_{1}(s) & G_{3}(s) \\
G_{2}(s) & G_{4}(s)
\end{array}\right]\left[\begin{array}{c}
u_{\mathrm{sc}}(s) \\
T_{\mathrm{Lc}}(s)
\end{array}\right],
\end{aligned}
$$




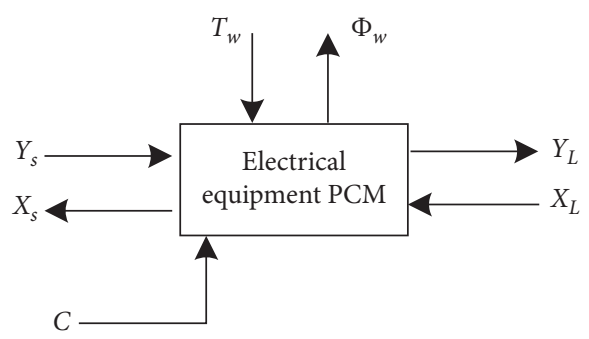

(a)

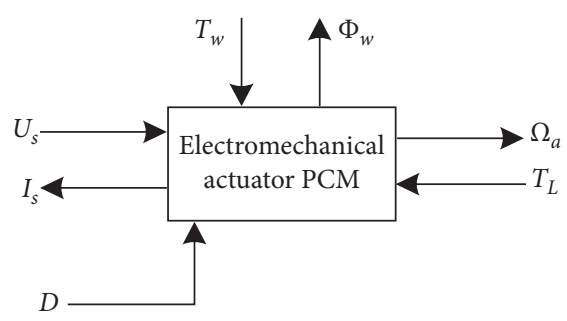

(b)

FIgURE 3: Input/output variables of the power transformation model. (a) Variable relationship of electrical equipment PCM. (b) Variable relationship of electromechanical actuator PCM.

where the voltage applied to the motor armature winding under the SCM control is $u_{\mathrm{sc}}$, and the relationship with the power source voltage $U_{s}$ is $u_{\mathrm{sc}}=\mathrm{DU}_{s} . T_{\mathrm{Lc}}$ is the load torque corresponding to the electromagnetic torque. In addition to the resistance torque $T_{L}$ on the rudder surface, it will also include the friction torque and damping torque on the motor and reducer.

3.4. Power Loss Model of PCM. The power loss model of equation (2) is generally described according to the power consumption principle of the device. For an electrical device without energy storage characteristics, the input power $P_{s}$ and the output power $P_{L}$ can also be approximated and described as follows:

$$
\Phi_{w}=P_{s}-P_{L}=Y_{s} X_{s}-Y_{L} X_{L}
$$

The electromechanical actuator shown in Figure 1 is also taken as an example. The power loss is mainly the inverter loss $\Delta P_{\mathrm{ec}}$, the copper loss $\Delta P_{\mathrm{cu}}$ of the motor, the iron loss $\Delta P_{\text {fe }}$ of the motor, the mechanical loss $\Delta P_{m}$ caused by the friction of the motor and the reducer, and the power loss of the electronic device which can be approximated to the motor copper loss $\Delta P_{\mathrm{cu}}$ and classified into the copper loss. Therefore, the power loss can be described as

$$
\begin{aligned}
\Phi_{w} & =\Delta P_{\Sigma}=\Delta P_{\mathrm{ec}} \Delta P_{\mathrm{cu}}+\Delta P_{\mathrm{fe}}+\Delta P_{m} \\
& =k_{\mathrm{ec}} I_{d}+k_{\mathrm{cu}} I_{d}^{2}+k_{\mathrm{fe}} \Omega_{m}+k_{m} \Omega_{m} \\
& =k_{\mathrm{ec}} I_{d}+k_{\mathrm{cu}} I_{d}^{2}+\left(k_{\mathrm{fe}}+k_{m}\right) \Omega_{m},
\end{aligned}
$$

where $I_{d}$ is the armature current. Due to the motor moment inertia, the electrical drive device is expressed as the device containing the energy storage component. If equation (6) is used to analyze the device loss, its dynamic characteristics will have some error.

\section{Information Conversion Module of Multiphysical Domain Model}

The SCM of the multiphysical domain model is used to simulate the information conversion of the electrical equipment. It can realize the operation control and the state measurement of the system, such as the functions of the controller and the sensor in Figure 1.
4.1. Control Information Conversion of SCM. For the multiphysical domain model described in Figure 2, the first function of the SCM is to simulate the electrical device control function, which forms the control signal $y_{\mathrm{sc}}$. The second function is to generate the disturbance signal $x_{\mathrm{Lc}}$ according to the operating state. It can be described as

$$
\left.\begin{array}{l}
y_{\mathrm{sc}}(t)=f_{\mathrm{sc}}\left(Y_{s}, X_{s}, Y_{L}, X_{L}, R^{*}, t\right) \\
x_{\mathrm{Lc}}(t)=f_{\mathrm{Lc}}\left(Y_{s}, X_{s}, Y_{L}, X_{L}, R^{*}, t\right)
\end{array}\right\},
$$

where $R *$ is the reference signal given by the host computer and other devices.

The electromechanical actuator shown in Figure 1 is still taken as an example. Since it is a position servo control system, the device needs to realize three closed-loop control of the rudder surface position $\theta_{a}$, the motor speed $\Omega_{m}$, and the motor armature current $I_{d}$. The control voltage signal should be

$$
\begin{aligned}
u_{\mathrm{sc}} & =\mathrm{DU}_{s}=W_{\mathrm{acr}}\left(I_{d}^{*}-I_{d}\right) U_{s} \\
& =W_{\mathrm{acr}}\left[W_{\mathrm{asr}}\left(\Omega_{m}^{*}-\Omega_{m}\right)-I_{d}\right] U_{s} \\
& =W_{\mathrm{acr}}\left\{W_{\mathrm{asr}}\left[W_{\mathrm{apr}}\left(\theta_{a}^{*}-\theta_{a}\right)-\Omega_{m}\right]-I_{d}\right\} U_{s},
\end{aligned}
$$

where $W_{\text {acr }}, W_{\text {asr }}$, and $W_{\text {apr }}$ are the transfer functions of current, speed, and position regulator, $I_{d} *$ is the reference value of the armature current, $\Omega_{m} *$ is the reference value of motor speed, and $\theta_{a} *$ is the reference value of the rudder surface position. The resistance torque applied to the aircraft rudder surface can be expressed by the hinge moment, and $k_{\theta}$ is the hinge moment coefficient of the rudder surface, which is $T_{L}=k_{\theta} \theta_{a}$. The load torque on the actuator is

$$
T_{\mathrm{Lc}}=T_{f}+k_{\Omega} \Omega_{m}+k_{\theta} \theta_{a},
$$

where $T_{f}$ is the friction torque of the motor and the reducer and $k_{\Omega}$ is the speed damping coefficient of the motor.

4.2. Test of Running Status. The function of the SCM operation state measurement is to simulate the sensor function of the electrical equipment. The test signal can be used as a feedback signal of the system control and provide to the monitoring device or the host computer, which is the output information in Figure 2.

The SCM test information is defined as $S$, which can be expressed as 


$$
S(t)=f_{s}\left(Y_{s}, X_{s}, Y_{L}, X_{L}, R^{*}, t\right) .
$$

For the EMA control and disturbance signals in equations (9) and (10), the required test signals are

$$
\left.\begin{array}{l}
I_{d}=k_{s} I_{s} \\
\Omega_{m}=k_{i} \Omega_{a} \\
\theta_{a}=\int_{0}^{t} \Omega_{a} \mathrm{~d} t
\end{array}\right\},
$$

where $I_{d}$ is the motor armature current, $I_{s}$ is the power supply current, $k_{s}$ is the proportional coefficient, $\Omega_{a}$ is the angular velocity of the aircraft rudder surface, $\Omega_{m}$ is the motor speed, $K_{i}$ is the reduction ratio of the reduction gear, and $\theta_{a}$ is the rudder surface position.

If it needs to know other signal information, such as the motor electromagnetic torque $T_{e}$, it can be described by

$$
T_{e}=k_{t} I_{d}=k_{t} k_{s} I_{s},
$$

where $k_{t}$ is the torque factor.

\section{Modeling and Verification of Electromechanical Actuator}

The electromechanical actuator is used as multiphysical domain modeling object, and the consistency is verified by comparison with the original model simulation results.

5.1. Electromechanical Actuator Parameters. The multiphysical domain modeling object is the EMA model shown in Figure 1. It is composed of nonlinear characteristic components such as the inverter and the brushless DC motor, which is called the EMA original model. For example, the main parameters of an EMA are the power supply voltage $270 \mathrm{VDC}$, the motor rated angular speed $600 \mathrm{rad} / \mathrm{s}$, the maximum rudder surface torque of $11500 \mathrm{~N} \cdot \mathrm{m}$, the electromagnetic time constant $0.000865 \mathrm{~s}$, and the electromechanical time constant 0.00652 s.

5.2. Electromechanical Actuator Modeling. The multiphysical domain modeling is mainly to establish the PCM transfer functions of $G_{1}(s), G_{2}(s), G_{3}(s), G_{4}(s)$ with equation (2) and the power loss with equation (4). According to the original model of the electromechanical actuator shown in Figure 1, the transfer function $G_{1}(s) \sim G_{4}$ $(s)$ with equation (5) can be established by the principle analysis method or the signal identification method.

Since the EMA in Figure 1 contains nonlinear components, the principle analysis modeling is complex; the signal identification method is adopted. The original model shown in Figure 1 is set as the open-loop structure. A step signal is applied to the control voltage $u_{\mathrm{sc}}$, and it is simulated with $T_{L}=0$ to obtain the unloaded angular velocity $\Omega_{a 0}$ of the rudder surface and the power supply current $I_{s 0}$. Then, the load torque $T_{L}$ is set to a step signal for simulation, and the speed signal $\Delta \Omega_{a}$ and the power source current $\Delta I_{s}$ are obtained. The obtained signals are, respectively, identified and the PCM is described as follows:

$$
\left.\begin{array}{l}
G_{1}(s)=\frac{\Omega_{m 0}(s)}{u_{\mathrm{sc}}(s)}=\frac{3.13}{7.33 \times 10^{-7} s^{2}+2.303 \times 10^{-3} s+1} \\
G_{2}(s)=\frac{I_{s 0}(s)}{u_{\mathrm{sc}}(s)}=\frac{6.69 \times 10^{-3} s+0.0005}{8.781 \times 10^{-7} s^{2}+2.404 \times 10^{-3} s+1} \\
G_{3}(s)=\frac{\Delta \Omega_{m}(s)}{T_{\mathrm{Lc}}(s)}=\frac{-1.24 \times 10^{-3} s-3.72}{7.618 \times 10^{-7} s^{2}+2.36 \times 10^{-3} s+1} \\
G_{4}(s)=\frac{\Delta I_{s}(s)}{T_{\mathrm{Lc}}(s)}=\frac{3.68}{8.578 \times 10^{-7} s^{2}+2.398 \times 10^{-3} s+1}
\end{array}\right\} .
$$

Combining the power conversion module of equation (14) with the information conversion module of equations (9) and (10), the multiphysical domain model shown in Figure 2 can be constructed.

5.3. Simulation Verification of Electromechanical Actuator. The reference position $\theta_{a} *$ of the actuator is set to $25^{\circ}$ in the simulation. The multiphysical domain model and the original model are simultaneously simulated and compared to obtain the EMA motion speed $\Omega_{a}$ (potential variable) and the power supply current $I_{s}$ (flow variable). The curves are shown in Figure 4. The dotted line is the simulation curve of the multiphysical domain model, and the solid line is the simulation curve of the original model.

It can be seen from Figure 4 that the actuator speed simulation curve of the multiphysical domain model is basically consistent with the original model, while the power supply current and the power loss have some errors in the subtleties, which is due to the model linearization. The error effect can be ignored for the simulation goal proposed in this paper.

\section{Integrated Method of Multiphysical Domain Model}

Another advantage of the multiphysical domain model is that it facilitates large-scale integration to establish the complex electrical system. The multiphysical domain model integration is based on the energy conversion relationship, and there are two types of series integration and parallel integration.

6.1. Serial Integration of Multiphysical Domain Model. The multiphysical domain model of complex electrical equipment can be obtained by the serial integration of multiple simple multiphysical domain models.

For example, the EHA consists of the motor, the hydraulic pump, and the hydraulic actuator. If the EHA original model is available, the same EMA signal identification modeling method as equation (14) can be used. If 


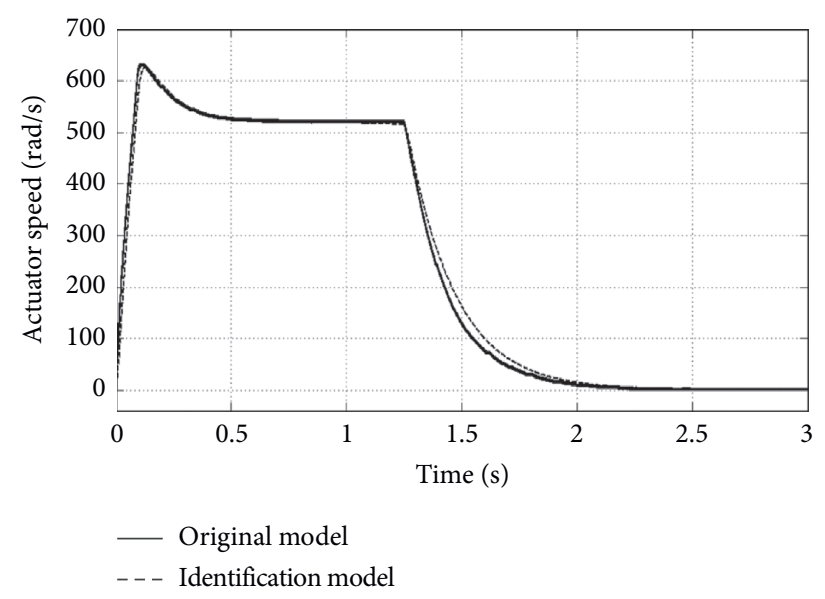

(a)

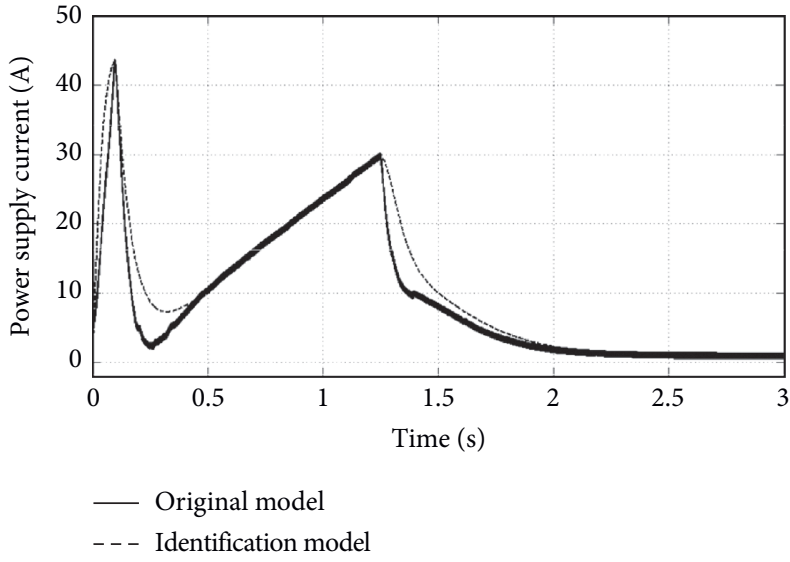

(b)

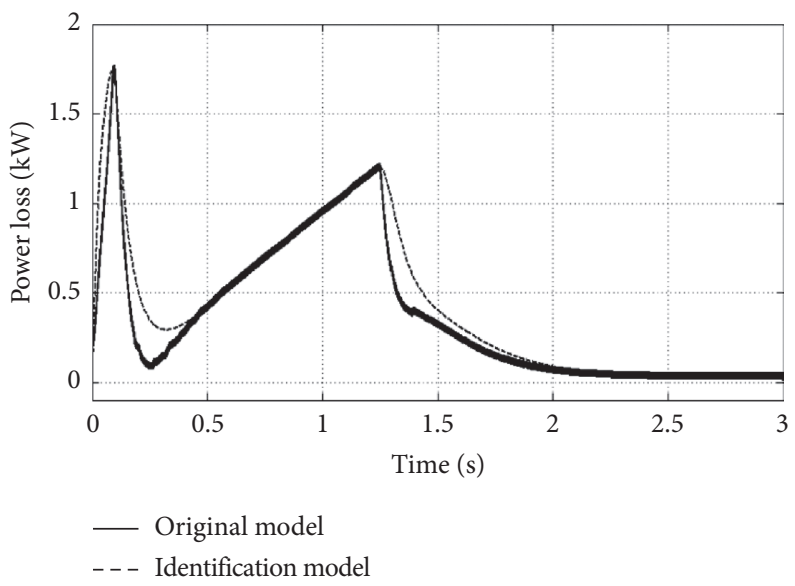

(c)

Figure 4: Simulation results of EMA models. (a) Actuator speed. (b) Power supply current. (c) Power loss.

there is no original model, it can also be modeled by the serial integration of the motor, the hydraulic pump, and the hydraulic actuator. In the integration, the consistency of the potential variable and the flow variable must be guaranteed. The structure is shown in Figure 5.

Since the EHA is controlled by the motor speed to realize the flow control of the hydraulic pump and the position control of the actuator, the control signal from the information conversion module is applied to the motor model. In addition, the EHA output is a linear motion; therefore, the potential variable of the output power according to Table 1 is the force $F_{a}$, and the flow variable is the moving speed $v_{a}$.

For example, the main parameters of the EHA are the power supply voltage $270 \mathrm{VDC}$, the motor rated speed $8000 \mathrm{r} / \mathrm{min}$, the actuator rated moving speed $0.12 \mathrm{~m} / \mathrm{s}$, and the maximum force $200 \mathrm{kN}$. The EHA model is simulated with the position which is set to $80 \mathrm{~mm}$. The simulation waveforms are shown in Figure 6. Among them, Figures $6(\mathrm{a}) \sim 6(\mathrm{~d})$ are the actuator output (mechanical energy), the hydraulic pump output (hydraulic energy), the motor output (mechanical energy), and the motor input (electrical energy). Figure 6(e) shows the power loss and the total loss of each EHA part.

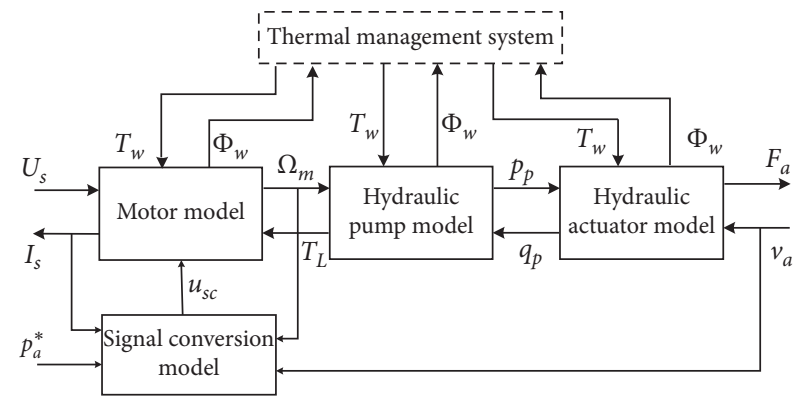

FIgURE 5: EHA model implemented by serial integration.

It can be seen from the simulation results that the EHA model obtained by the series integration of the multiphysical domain model can not only simulate the EHA input and output variables and power data but also observe the waveforms of each connection point in order to analyze the correctness of the model.

6.2. Parallel Integration of Multiphysical Domain Model. In the electrical system of the more electric aircraft, the power supply needs to provide electrical power for all the 


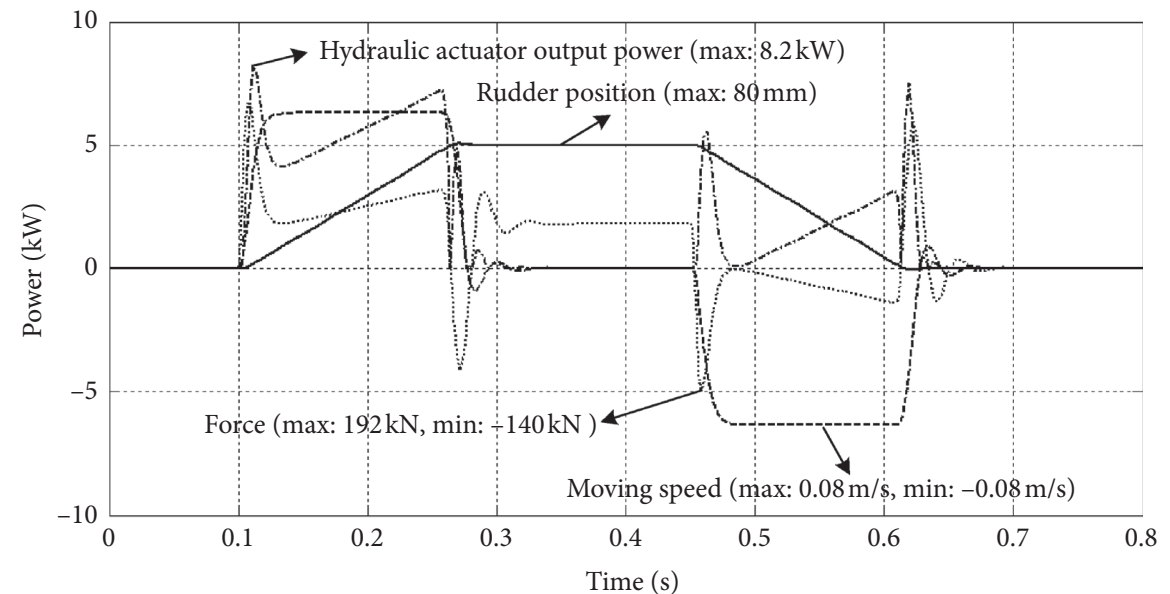

(a)

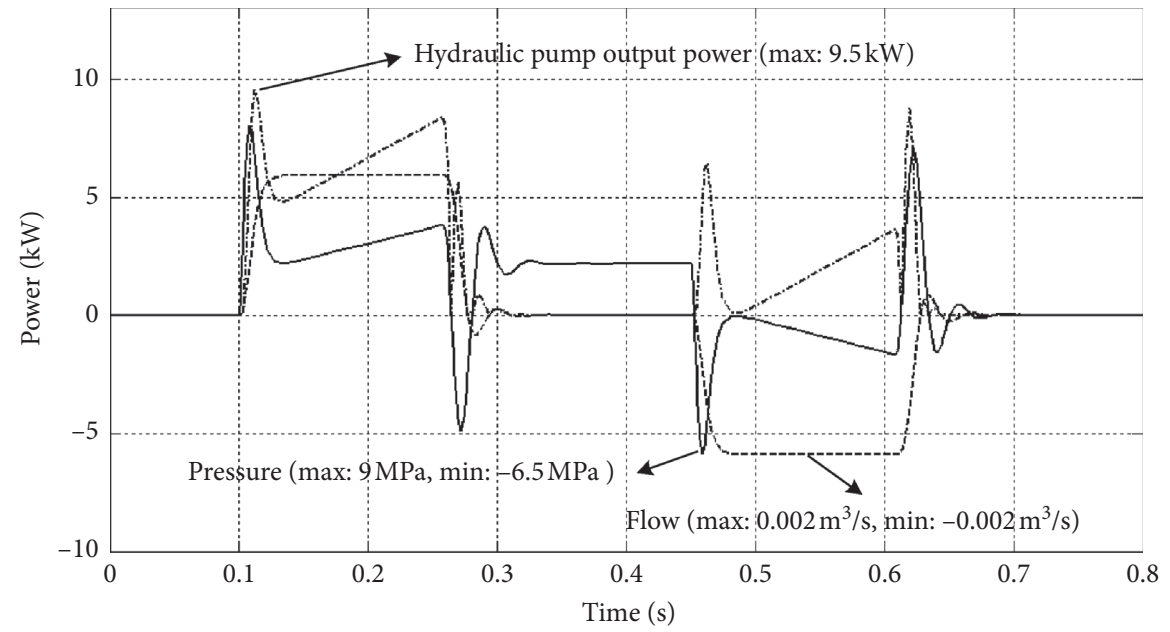

(b)

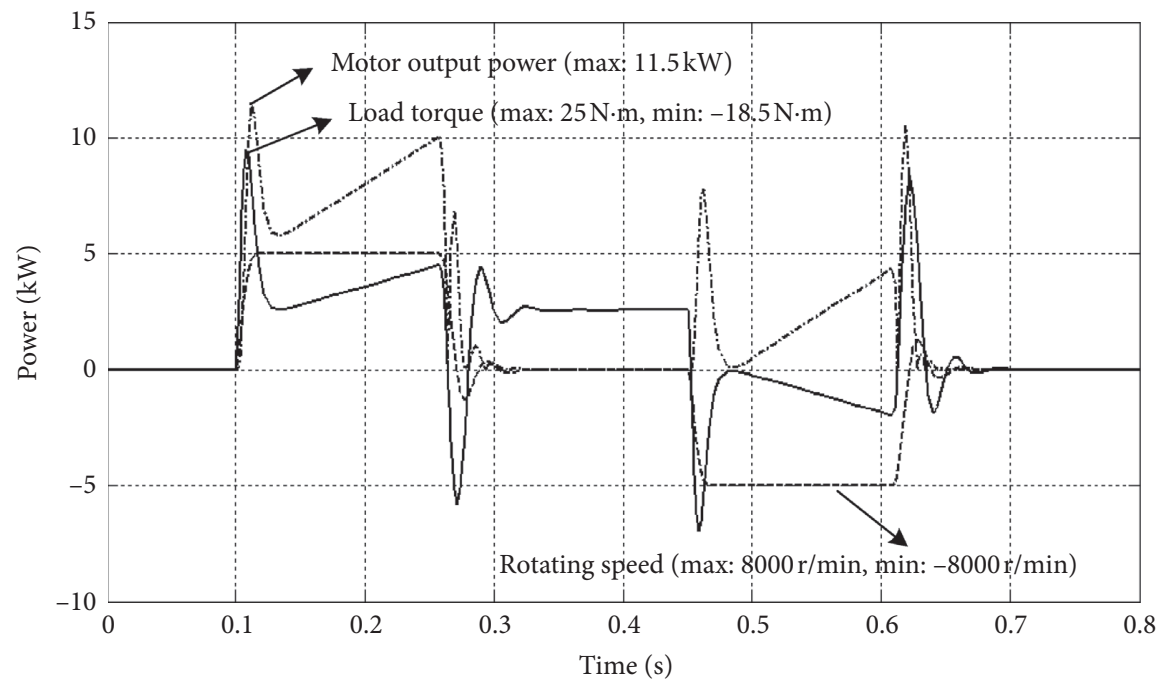

(c)

Figure 6: Continued. 


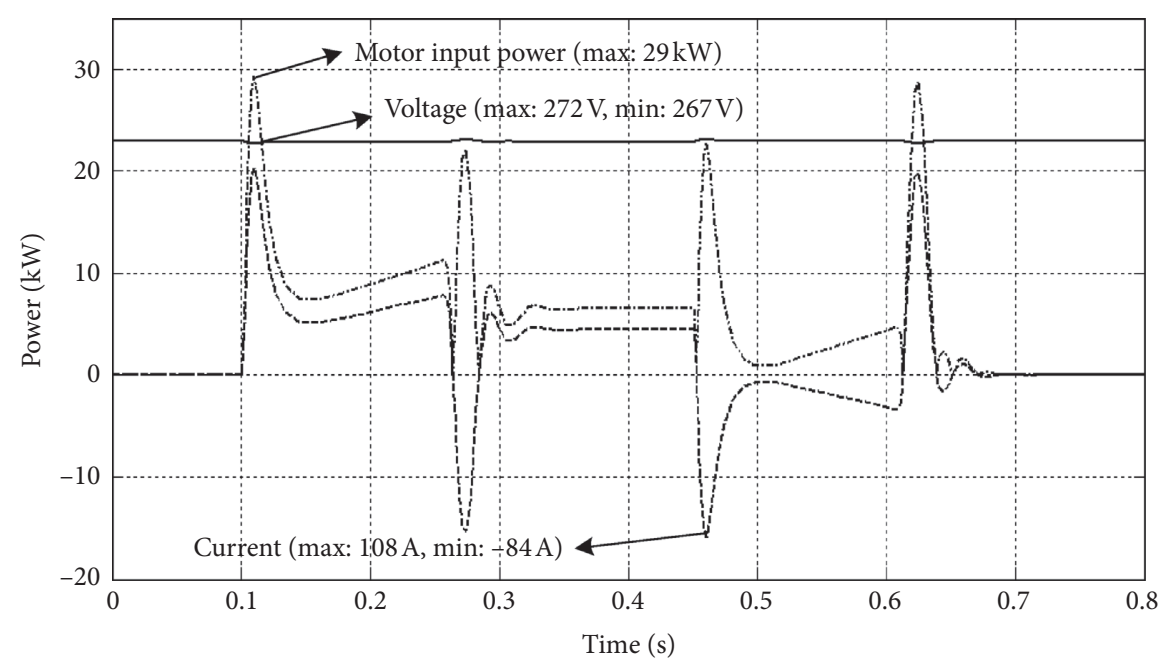

(d)
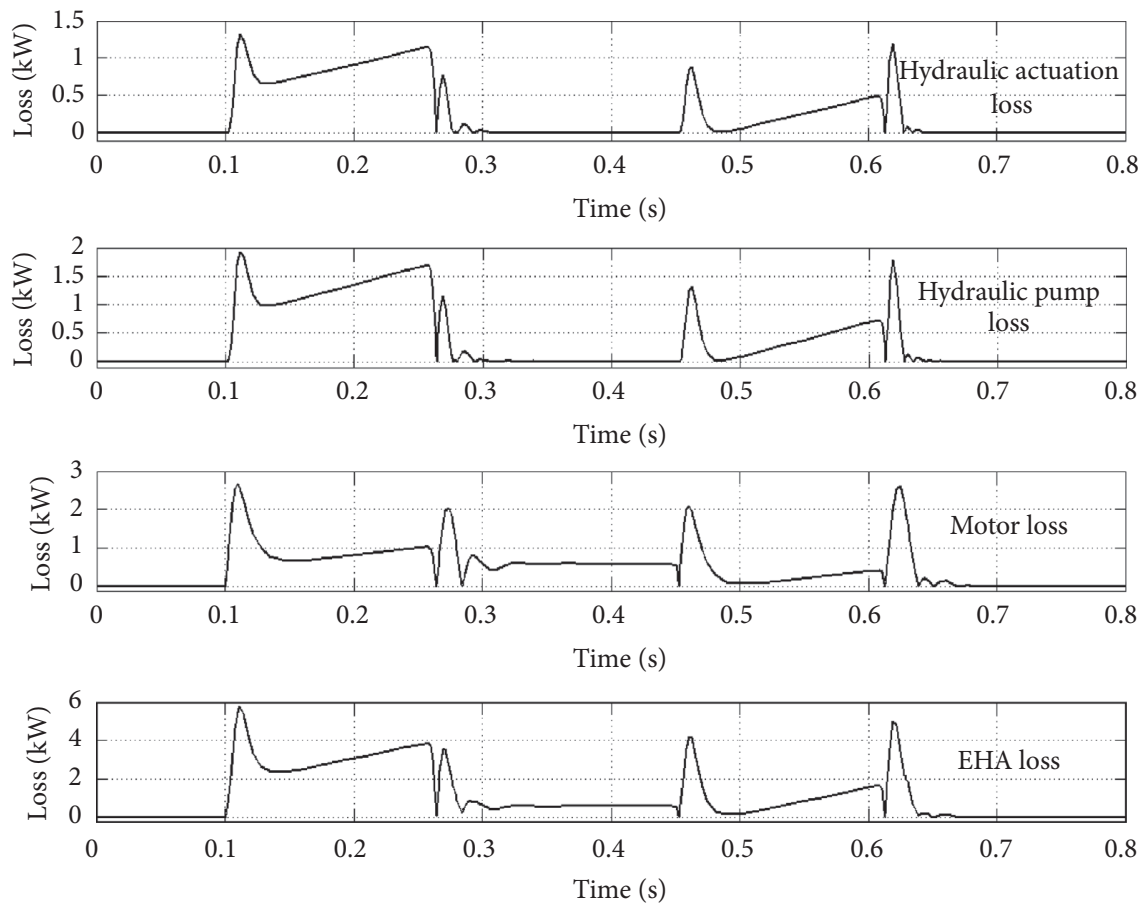

(e)

Figure 6: Simulation waveforms of each EHA connection point. (a) Moving position, power, and variables of the rudder surface. (b) Hydraulic power and variables of the hydraulic pump. (c) Mechanical power and variables of the motor. (d) Electrical power and variables of the motor. (e) Power loss of EHA.

electrical equipment; that is, the electrical equipment forms the parallel structure on the bus bar. When all the electrical equipment is described by the multiphysical domain models, the structure is as shown in Figure 7.

The output power of the power supply is the electrical energy. The potential variable is the power supply voltage $U_{s}$ connected to the potential variable $U_{\mathrm{si}}$ of all electrical loads with the power supply side. The flow variable $I_{s}$ is the summation current of the electrical equipment input current, which is the power flow $I_{\mathrm{si}}$ summation of all electrical loads.

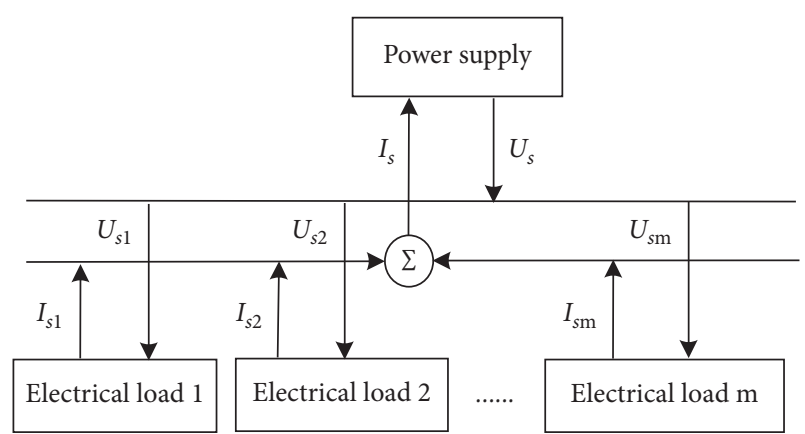

FIGURE 7: Parallel integration of the power supply system. 


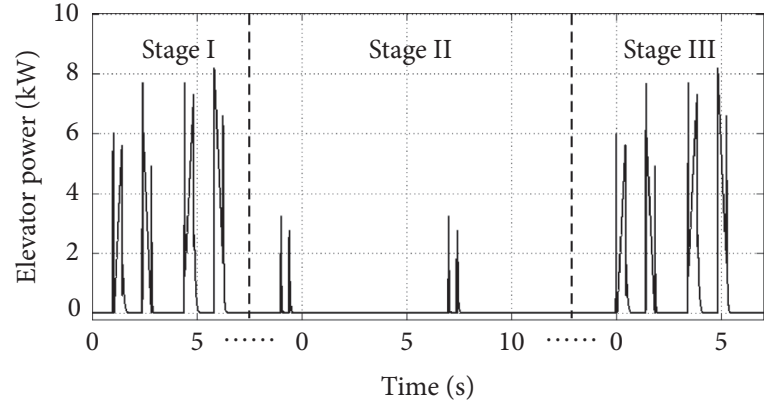

(a)

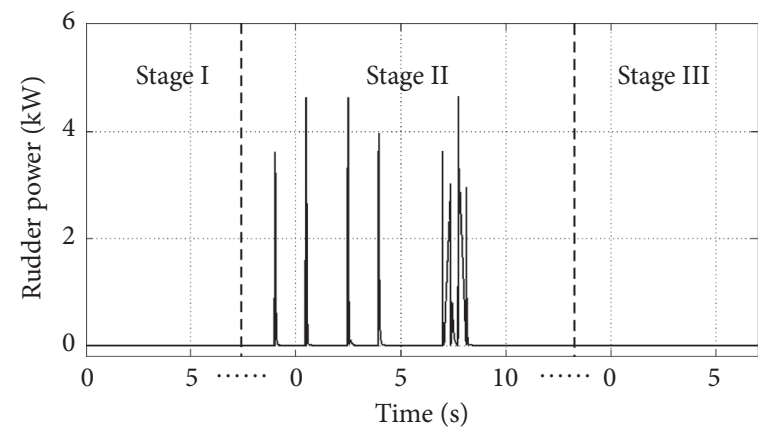

(c)

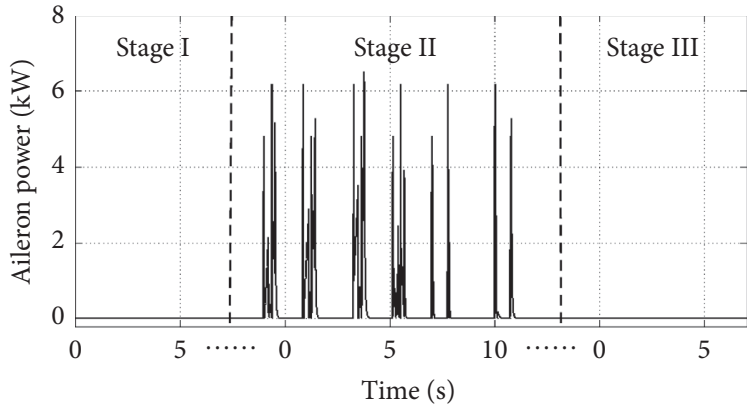

(b)

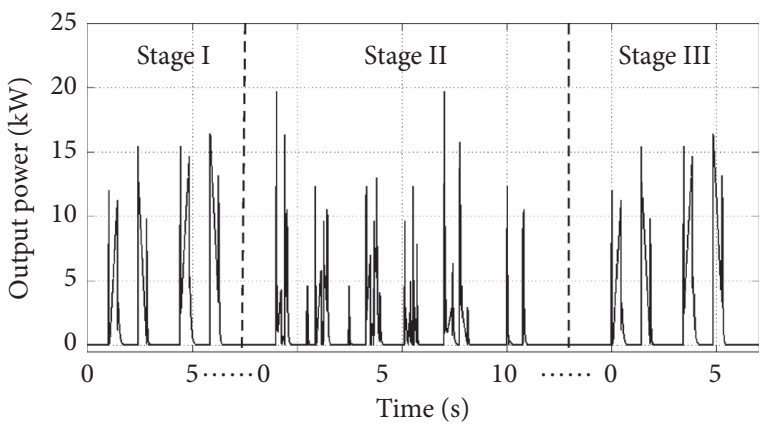

(d)

Figure 8: Flight characteristics simulation of the flight control system. (a) Elevator power characteristics. (b) Aileron power characteristics. (c) Rudder power characteristics. (d) Output power characteristics of the power supply.

$$
\left.\begin{array}{l}
U_{s}=U_{s 1}=U_{s 2}=\cdots=U_{s \mathrm{sm}} \\
I_{s}=I_{s 1}+I_{s 2}+\cdots+I_{s m}
\end{array}\right\},
$$

The flight control system of the aircraft is taken as an example. The flight control system consists of the left and right ailerons, left and right elevators, and the rudder. It is assumed to be driven by the EMA, established with the power supply system shown in Figure 7 and simulated.

It is assumed that the aircraft continuously completes the climb phase, the combat phase (including the yaw, roll, and dive flight attitude), and the descent phase of the flight. The control signals are obtained by the flight control system models. The corresponding reference signal $\theta_{a} *$ is set for each EMA. The power characteristics of the simulation are shown in Figure 8. Figures $8(a) 8(c)$ are the power characteristics of the elevator, the aileron, and the rudder, respectively. Figure $8(\mathrm{~d})$ shows the power characteristics of the power supply. The movements of the left and right elevators and ailerons are symmetrical; therefore, the power characteristics are the same.

It can be seen from the simulation results shown in Figure 8 that the elevator is the main drive device when the aircraft is climbing and descending. The elevator, aileron, and rudder need to be integrated operation when the aircraft is rolling and yawing, and the power conversion is complicated.

Since the electromechanical actuator belongs to the dynamic load of the short-time operation, the flight control system shows the pulse power of different sizes from the above figures. The power analysis for this type of system can only be done by the digital simulation.

\section{Conclusion}

The multiphysical domain model with the parameter identification according to the original model data is applied in this paper. It takes the power conversion process of the electrical equipment as the basic modeling and accurately expresses the power conversion and loss characteristics based on the dynamic characteristics of the device itself. The model based on the energy conversion relationship for system integration is to achieve the large-scale integration of the electrical system flexibly and the required digital simulation of the electrical system design.

\section{Data Availability}

The data used to support the findings of this study are available from the corresponding author upon request.

\section{Conflicts of Interest}

The authors declare that they have no conflicts of interest.

\section{References}

[1] J. A. Rosero, J. A. Ortega, E. Aldabas, and L. Romeral, "Moving towards a more electric aircraft," IEEE Aerospace and Electronic Systems Magazine, vol. 22, no. 3, pp. 3-9, 2007.

[2] R. I. Jones, "The more electric aircraft-assessing the benefits," Proceedings of the Institution of Mechanical Engineers, Part G: Journal of Aerospace Engineering, vol. 216, no. 5, pp. 259-269, 2002. 
[3] P. Wheeler, "Technology for the more and all electric aircraft of the future," in Proceedings of the International Conference on Automatic, Curico, Chile, October 2016.

[4] C. R. Avery, S. G. Burrow, and P. H. Mellor, "Electrical generation and distribution for the more electric aircraft," in Proceedings of the 42th International Universalities Power Engineering Conference, pp. 1007-1012, Brighton, UK, September 2007.

[5] Y. Ji and M. Kuhn, "Model based system level studies of more electric aircraft," in Proceedings of Aerospace Systems and Technology Conference, Cincinnati, OH, USA, September 2014.

[6] J. C. Shaw, S. D. A. Fletcher, P. J. Norman, and S. J. Galloway, "More electric power system concepts for an environmentally responsible aircraft," in Proceedings of the 47th International Universities Power Engineering Conference, pp. 1-6, London, UK, April 2012.

[7] T. O'connell, G. Russell, and K. Mccarthy, "Energy management of an aircraft electrical system," in Proceedings of the 46th AIAA/ASME/SAE/ASEE Joint Propulsion Conference \& Exhibit, Nashville, TN, USA, July 2010.

[8] J. Nairus and R. Fingers, More Electric Aircraft Power and Thermal Management, Technologies for Energy Optimized Aircraft Equipment Systems Forum Paris, France, 2006.

[9] F. Schettni, E. Denti, and G. D. Rito, "Development of a simulation platform of all electric aircraft on-board system for energy management studies," Aeronautical Journal, vol. 121, no. 1239, pp. 710-719, 2017.

[10] A. M. Law, T. Y. Xiao, and W. H. Fan translated, Simulation Modeling and Analysis, Qinghua University, Beijing, China, 4th edition, 2012.

[11] E. Walters, M. Amrhein, and T. O'connel, "INVENT modeling, simulation, analysis and optimization," in Proceedings of the 48th Aiaa Aerospace Sciences Meeting Including the New Horizons Forum and Aerospace Exposition, pp. 1-10, Orlando, FL, USA, January 2010.

[12] J. Zhong, Coupling Design Theory and Method of Complex Electromechanical System, Mechanical press, Beijing, China, 1 st edition, 2007.

[13] R. Geoff, K. Nick, and B. Steve, "Bond graph modeling of a hydraulic vibration system: simulation and control," in Proceedings of the Integrated Modeling and Analysis in Applied Control and Automation, pp. 281-286, Rome, Italy, September 2011.

[14] M. Mutuku and K. John, "Bond graph modeling of interactuator in teractions in a multi-cylinder hydraulicsystem," World Academy of Science," Engineering and Technology, vol. 74, pp. 32-41, 2011.

[15] B. Li, W. Li, and X. Zhang, "Modeling and simulation of aircraft power supply system based on Dymola and Modelica," ", in Proceedings of the Electrical Systems for Aircraft, Railway, Ship Propulsion and Road Vehicles \& International Transportation Electrification Conference, Toulouse, France, November 2017.

[16] J. Bals, Y. Ji, and M. Kuhn, "Model based design and integration of more electric aircraft systems using modelica," in Proceedings of the European Power Electronics Conference and Exhibition, pp. 1-13, Europe, Spain, September 2009.

[17] L. Liu, Z. Cao, and L. R. Sun, "Energy optimization characteristic analysis of electromechanical actuator on more electric aircraft," in Proceedings of the Communications in Computer and Information Science, pp. 41-52, Beijing, China, June 2016.

[18] G. Qiao, G. Liu, Z. Shi, Y. Wang, S. Ma, and T. C. Lim, "A review of electromechanical actuators for More/All Electric aircraft systems," Journal of Mechanical Engineering Science, vol. 232, no. 22, pp. 4128-4151, 2018.

[19] G. D. Rito, R. Galatolo, and F. Schettini, "Experimental and simulation study of the dynamics of an electro-mechanical landing gear actuator," in Proceedings of the 30th International Council of the Aeronautical Sciences, Daejeon, South Korea, September 2016.

[20] W. N. Hu, L. Zhou, and Y. S. Tian, "Analysis for the power loss of electrohydrostatic actuator and hydraulic actuator," in Proceedings of the Advanced Intelligent Mechatronics, pp. 613-616, Busan, South Korea, July 2015.

[21] Y. Li, Z. Ji, L. Yang, P. Zhang, B. Xu, and J. Zhang, "Thermalfluid-structure coupling analysis for valve plate friction pair of axial piston pump in electrohydrostatic actuator (EHA) of aircraft," Applied Mathematical Modelling, vol. 47, pp. 839858, 2017. 